\title{
Review of: "Metabolic-scale gene activation screens identify SLCO2B1 as a heme transporter that enhances cellular iron availability"
}

\author{
McKale Montgomery ${ }^{1}$ \\ 1 Oklahoma State University
}

Potential competing interests: The author(s) declared that no potential competing interests exist.

\section{Summary}

This work provides evidence that induction of SLCO2B1 expression may enhance cellular iron availability and protect against iron restriction. However, lack of obvious changes in band density, data quantitation and proper statistical analyses fail to support many of the conclusions drawn. Also, the use of lysosomal inhibitors to indirectly restrict iron access without including essential positive controls that demonstrate the cells were experiencing reduced iron availability weaken some of the authors' claims Major Concerns

1. Typically, cells can handle modest levels of iron restriction, so the findings that cell proliferation is not at all impacted by $0.75 \mu \mathrm{M}$ DFO treatment, but that proliferation is nearly abolished by $1.5 \mu \mathrm{M}$ treatment seems unusual. Iron is often limiting in cell culture conditions, so it is important to make sure cultured cells are iron replete before performing iron restriction studies.

2. How long were the cells treated with $2 \mu \mathrm{M}$ DFO and $5 \mathrm{nM}$ BafA1? According to the results in Figure 1G, vast majority of cells would be dead after 5 days of treatment with these doses, and gene profiling dead cells provides limited information. Were the cells really iron deficient or just dying? It seems if the cells were iron deficient, changes in canonical iron related genes (e.g., TFRC) would have been detected.

3. Western blots should be quantitated, and statistical analyses should be performed. This would address many of the following comments/concerns.

1. Figure $2 \mathrm{G}$ - There needs to be an explanation as to why/how BafAl and $\mathrm{NH} 4 \mathrm{Cl}$ treatment alone somehow appears to increase the expression of both IRP2 AND FTH1 in Jurkat cells compared to control Jurkat cell. The induction of IRP2 protein expression with BafA1 and $\mathrm{NH} 4 \mathrm{Cl}$ treatment makes biologic sense along with the decrease in FTH1 expression as occurs in the 239T cells, so the authors should address this.

2. Moreover, the authors state that SLCO2B1 expression "strongly blocked the increase in IRP2 and decrease in $\mathrm{FTH} 1$ protein levels in response to treatment with iron chelators or lysosomal $\mathrm{pH}$ inhibitors." Yet, there appears to be no difference in IRP2 expression in the DFO treated Jurkat or 293T cells, and FTH1 protein was not even detected in DFO treated 293T cells. This suggests that SLCO2B1 is sustaining cell proliferation in response to iron chelation and lysosomal pH inhibition by 
entirely different mechanisms.

3. Also, IRP2 can be regulated independently of iron (e.g. by ROS and hypoxia), and the authors have not demonstrated that any of their treatments caused overt iron deficiency. Reduced cellular iron levels or appropriate directional changes in TFRC and/or FTH1 protein levels could be used to address this concern. The authors use FTH1 in Figure 1G, but FTH1 protein expression goes from nothing to nothing in the Jurakt cells following DFO treatment, and as mentioned above, FTH1 expression actually appears to increase compared to control cells with BafAl and $\mathrm{NH}_{4} \mathrm{Cl}$ treatment.

4. Figure 2J - the authors say SLCO2B1 expression "completely restored" cell survival and proliferation in response to TFRC loss, but the graph does not support this statement, and it does not look like those statistical comparisons to the control vector cells were actually run.

5. Figure 3 - the authors are trying to determine what is essential for SLCO2B1-mediated resistance to "iron restriction" yet choose a messy lysosomal inhibitor for these analyses rather than a cleaner method of iron restriction. Indeed, iron metabolism genes appear largely unaffected. To demonstrate iron-specific results, control experiments using iron-specific chelators, or actual iron supplementation and then iron restriction should have been included.

6. In Figure 4J the authors claim that "hemin treatment resulted in greater reduction of ALAS1 levels in SLCO2B1-expressing cells." Yet, ALAS1 was not even detected in control cells in the top set of bands, and the bands indicating ALAS1 in the untreated and hemin treated samples in the second row look identical.

Minor Concerns:

1. Throughout the paper the authors present treatment-induced changes in cell proliferation as a reduced number of total cell doublings over time. However, these "doublings" were assessed using a kit that measures ATP production. As iron restriction can significantly reduce TCA cycle and ETC flux, and subsequently ATP production, lower ATP levels do not necessarily mean less doublings but could just indicated lower energy production in iron deficient cells. In the presented format, it is also not clear what negative number of cell doublings mean. Cell death?

2. The quantitation of heme versus non-heme iron in control versus SLCO2B1 expressing cells instead of just total iron would strengthen the evidence that SLCO2B1 rescues cells from iron restriction by specifically increasing heme iron uptake.

3. The rationale for the time discrepancies ( 24 hours for Jurkat cells versus 15 minutes for HepG 2 and PaTu-8988t cells) of ZnMP treatments between cell lines in Figures 4 B-D should be explained.

4. Figures $4 \mathrm{E}$ and $\mathrm{F}$ are missing some error bars

5. The abbreviations I.e. and h.e. in figure $4 \mathrm{~J}$ need to be defined. 